\title{
Canavan Disease - A Rare Case Report
}

\author{
Zahoor Hussain Daraz ${ }^{1}$, Berkheez Shabir ${ }^{2}$, Rehana Afshan ${ }^{3}$, Durri Shahwar ${ }^{4}$
}

\author{
${ }^{1}$ Senior Resident, Department of Paediatrics, Govt. Medical College, Doda, J\&K, India \\ \& Ex-Consultant of Paediatrics in Ministry of Health, Maldives \\ ${ }^{2}$ Senior Resident, Department of Obs-Gyne, Govt. Medical College, Doda, J\&K, India \\ \& Ex-Consultant of Obs-Gyne in Ministry of Health, Maldives \\ ${ }^{3}$ Consultant Radiologist, Noora Hospital, HMT, Srinagar, Jammu \& Kashmir, India \\ ${ }^{4}$ Assistant Professor, Department of Physics, Vishwa Bharti Women’s College, \\ Rainawari, Srinagar, Jammu \& Kashmir, India \\ Corresponding Author: Zahoor Hussain Daraz
}

\begin{abstract}
Canavan disease is a rare autosomal recessive disorder characterized by progressive leukodystrophy involving white matter of the brain. Disease leads to severe psychomotor retardation, seizures and premature death. More prevalent among Jewish population. Among non-Jewish population incidence is approximately 1:100000. Prognosis is guarded with current management only symptomatic and supportive. There is no effective treatment, however early gene therapy has improved the quality of life of patients. Furthermore, Lithium citrate has also shown certain positive results in experimental models using rats. Even few human studies have also been done, however promising results require larger controlled trials.
\end{abstract}

Key words: MRI: Magnetic Resonance and Imaging, EEG: Electro Encephalogram, CD: Canavan Disease, ASPA: acetyl aspartate, LUCS: Lower Uterine Cesarean Section, OFC: Occipito Frontal Circumference, NAA: Nacetyl aspartate

\section{BACKGROUND}

Canavan disease or Canavan-Van Bogaert-Bertrand disease is a fatal gene disorder resulting due to point mutation involving ASPA gene located on the short arm of chromosome 17p13.3. Frequently observed point mutations are p.Glu285Ala and p.Tyr231X. ${ }^{1}$ Patients usually undergo immense investigation before reaching to such diagnosis. It results due to deficiency of an enzyme aspartoacyclase that leads to the accumulation of $\mathrm{N}$-acetyl aspartate causing under-production of acetate and thereby interfering myelin sheath production. ${ }^{2}$ Patients in neonatal period have varied presentation such as delayed achievements of motor and mental milestones, macrocephaly and asymmetry of the palpebral fissure.

\section{CASE PRESENTATION}

"A" a 6 Months old, male infant was born by LUCS at 38 weeks of gestation through a consanguineous marriage, $2^{\text {nd }}$ in birth order, with an Apgar score of 8 and 10 at 1 and 5 minutes respectively. $1^{\text {st }}$ baby was born at term, died at 5 days of age probably due to fulminant sepsis and hypoglycemia. Baby had event free neonatal period. OFC was approximately $40 \mathrm{~cm}$ that falls on $97^{\text {th }}$ centile. Palpebral fissures were asymmetrical and mother noticed difficulty in breast feeding due to poor suck and swallow reflexes for which septic screening was done which was negative. Length of the baby was normal. Baby presented again to our hospital at 4 months of age with active seizures, lethargy, poor feeding and delayed achievement of motor mile stones.(Fig I) He was not able to hold his head yet and eye contact was also absent (visual impairment). Generalized hypotonia was found with transient spasticity. Baby was thoroughly examined and investigated. Septic screening 
was negative and electrolytes were within normal limits. EEG revealed generalized spikes or sharp waves, $3-\mathrm{Hz}$ or faster spikewave complexes. MRI of brain was performed revealing diffuse, symmetrical white matter degeneration in the subcortical areas, with bilateral involvement of the Globus pallidus. Gas chromatography mass spectroscopy showed increased acid $\mathrm{N}$ acetylaspartate which confirmed the diagnosis. Genetic analysis could not be performed due to financial constraints of the patient. Considering progressive macrocephaly, delayed motor mile stones and other features of neurological deterioration and increased $\mathrm{N}$ acetylaspartate in urine, diagnosis of Canavan disease was made. In order to improve the quality of life, symptomatic and early supportive therapy was implemented and thorough genetic family counselling was also done. ${ }^{3}$

\section{Patient consent Obtained.}

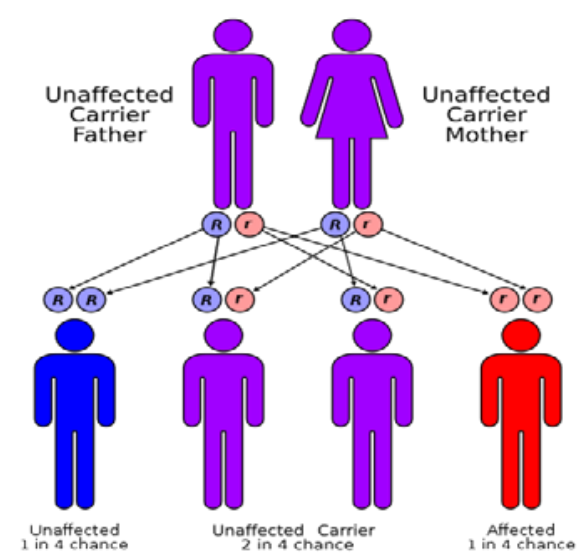

Fig I. Showing autosomal recessive inheritance character of the disease (Pic Courtesy internet)

\section{DISCUSSION}

Similar cases of neonatal CD have been reported in the literature where patients presented with lack of head control, macrocephaly and nystagmus between 4 and6 months of life. Urinalysis for estimating concentration of NAA with the help of gas chromatographic mass spectroscopy remains essential to diagnosis. Genetic testing confirms the diagnosis. The only currently available therapy is supportive care. Recent advances in medical

\section{TREATMENT}

Currently symptomatic and supportive treatment is the only therapy available. Gene therapy demonstrated certain promising results however need larger controlled trials. Discussing outcome of disease with parents, CD is characterized by hypotonia during neonatal days of life that changes into spasticity and failure of achievement of motor mile stones like lack of head control, sitting without support and delayed speech. Neck control delay is noticed after 3 months and macrocephaly is also observed with developmental delay by 5 months. Management is aimed at patient and family both. Where patient is given anti epileptics for seizure control, adequate nutrition and hydration for healthy life and prevention of infections. Family is counselled for future pregnancies. Prognosis is explained and is guarded with final outcome as death by teenage.

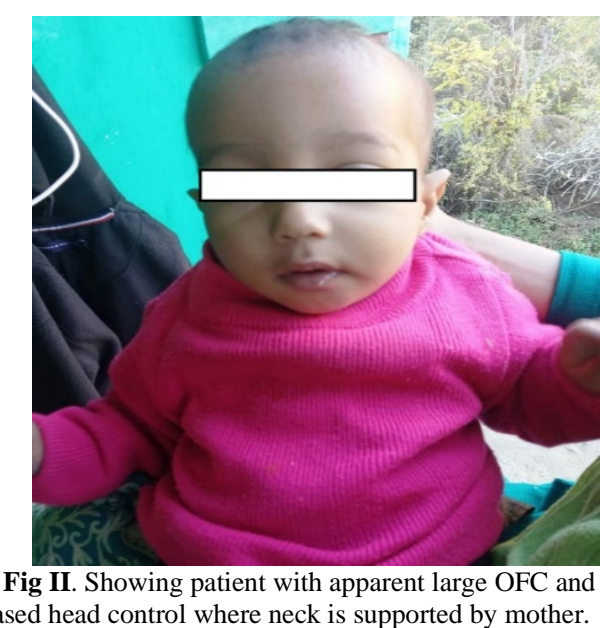

sciences have seen gene therapy being experimented achieving better results when therapy was started between 0 and 3 months of life. Adeno-associated viral vector carrying the ASPA gene (AAV2-ASPA) was used in gene therapy. The preliminary results were encouraging and significant decrease in elevated NAA in the brain was seen and progression of brain atrophy was seen to have slowed down. Patients were seen to have improved clinical status after treatment. ${ }^{4}$ Experimental treatment using 
lithium citrate in a rat genetic model of Canavan disease was able to significantly decrease levels of $\mathrm{N}$-acetyl aspartate. However when tested on a human, the patient's condition reversed during a 2-week wash-out period after withdrawal of lithium. $^{5}$ As Canavan disease has an autosomal recessive pattern of inheritance, family counselling has an important role in management of future pregnancies.(Fig: I1)

\section{Acknowledgement: None}

\section{Conflict of Interest: None}

\section{Source of Funding: None}

\section{REFERENCES}

1. Matalon, Reuben; Delgado, Lisvania; Michals-Matalon, Kimberlee (1993), Adam, Margaret P.; Ardinger, Holly H.; Pagon, Roberta A.; Wallace, Stephanie E. (eds.), "Canavan Disease", GeneReviews ${ }^{\circledR}$, Seattle (WA): University of Washington,
Seattle, PMID 20301412, ～retrieved 202109-10 2.

2. Boughamoura L, Chaabane F, Tilouche S, et al. [Canavan disease or N-acetyl asparticaciduria: a case report].Arch Pediatr 2007; 14:173-6.

3. Sreenivasan P, Purushothaman KK. Radiological clue to diagnosis of Canavan disease. Indian J Pediatr 2013; 80:75-7.

4. Leone P, Shera D, McPhee SW, et al. Longterm follow-up after gene therapy for Canavan disease. Sci Transl Med 2012.

5. Assadi, M.; Janson, C.; Wang, D. J.; Goldfarb, O.; Suri, N.; Bilaniuk, L.; Leone, P. (Jul 2010). "Lithium citrate reduces excessive intra-cerebral $\mathrm{N}$-acetyl aspartate in Canavan disease". European Journal of Paediatric Neurology. 14 (4): 354-359. doi:10.1016/ j.ejpn.2009.11.006.

How to cite this article: Daraz ZH, Shabir B, Afshan $\mathrm{R}$ et.al. Canavan disease - a rare case report. International Journal of Science \& Healthcare Research. 2021; 6(4): 348-350. DOI: https://doi.org/10.52403/ijshr.20211048 